\title{
Analyse morphométrique semi quantitative de l'histologie testiculaire au cours du vieillissement
}

\author{
Lucia BICCHIERAY ${ }^{1}$, Imen BEN FTIMA ${ }^{1}$, Martine ALBERT ${ }^{1}$, Marianne BERGERE ${ }^{1}$, \\ Olivier CUSSENOT ${ }^{2}$, Nicole PARSEGHIAN ${ }^{1}$, Mbarka DAKOUANE ${ }^{1}$, Jacqueline SELVA ${ }^{1}$. \\ 1 Laboratoire d'Embryologie, UFR Biomédicale des Saints-Pères, Paris \\ 2 Centre de Recherche sur la Pathologie Prostatique E 9 3104, Génopole, Evry
}

\begin{abstract}
RESUME
Les analyses macroscopiques, histologiques et morphomètriques du tissu testiculaire, effectuées sur des sujets âgés de 53 à 102 ans et ayant fait don de leur corps au Centre des Saints-Pères, montrent une grande variabilité individuelle, avec de très sévères altérations de la spermatogenèse dès 65 ans ou au contraire une spermatogenèse conservée jusqu'à l'âge de 95 ans. Les anomalies morphologiques détectées au niveau du testicule se manifestent principalement par une diminution du nombre des cellules de Sertoli et des cellules germinales, et par une augmentation de l'épaisseur de la membrane propre. Les cellules de Leydig s'hypertrophient avec l'âge, mais peuvent disparaître chez les sujets les plus âgés. Néanmoins, 19 des 39 sujets âgés de plus de 60 ans avaient une spermatogenèse complète et des spermatozoïdes présents dans certains tubes. Deux questions restent posées, celle des bases (génétiques et/ou environnementales) de cette variabilité individuelle et celle des accidents génétiques qui peuvent survenir au cours des méioses de ces hommes très âgés.
\end{abstract}

Mots clés : testicule, cellules germinales, membrane propre, épithélium séminifère, âge

\section{INTRODUCTION}

Poser la question de l'influence de l'âge de l'homme sur la fertilité n'est pas nouveau [24]. L'homme peut être père jusqu'à un âge avancé, et nous avons quelques exemples célèbres. Cependant, en dehors des études concernant la fécondance du sperme de l'homme âgé de plus de quarante ans $[3,9,19]$, quelques travaux ont permis de préciser l'influence du vieillissement sur l'histologie testiculaire, mais cette évolution n'a pas été observée jusqu'à un âge très avancé.

Un des premiers signes décrits est la sclérose artériolaire provoquant une ischémie progressive du tissu testiculaire [20, 23, 25]. Des signes de dégénérescence des cellules de Leydig ont été observés parallèlement à une baisse de testostérone plasmatique $[10,14,16,21]$. Au niveau des tubes séminifères plusieurs signes ont été décrits : un épaississement de la membrane propre [15], des anomalies des cellules de Sertoli qui paraissent être les dernières à disparaître $[13,15]$, et enfin, en ce qui concerne la lignée germinale, une perte progressive des cellules commençant par les spermatozoïdes et les spermatides avec un arrêt fréquent au stade pachytène $[2,4,12]$.

Jusqu'à présent les études publiées concernent un effectif réduit de patients et beaucoup se sont focalisées sur l'effet de l'âge sur un type cellulaire précis. Notre but a été d'effectuer une étude de l'ensemble des constituants du parenchyme testiculaire sur une série comportant des sujets d'âge extrêmement avancé.

Correspondance :

Dr Lucia BICCHIERAY Service d'Histologie Embryologie Cytogénétique, Centre Hospitalier Intercommunal de PoissySaint Germain en Laye, 10 rue du Champ Gaillard, 78303

Poissy Cedex - Tel 01.39.27.51.55 - Fax 01.39.27.44.25

Email ampcyto_poissy@hotmail.com 


\section{MATERIELS ET METHODES}

L'étude porte sur 8 témoins et 39 hommes décédés, âgés de 53 à 102 ans, ayant fait don de leur corps au Centre de l'Institut d'Anatomie des Saints Pères dans le cadre de l'enseignement et de la recherche scientifique. Dans tous les cas d'hommes âgés, les prélèvements ont été réalisés sur des sujets décédés dans les 24 heures précédant l'autopsie et le tissu testiculaire était de ce fait correctement conservé. De plus, des examens témoins de biopsie ont été réalisés. Ces biopsies proviennent d'hommes atteints d'azoospermie excrétoire (obstruction post-infection ou agénésie bilatérale des déférents) d'âge compris entre 29 et 42 ans (moyenne 35 ans) et consultant pour infertilité. Pour chaque sujet, nous avons réalisé successivement les trois études suivantes :

\section{Etude macroscopique}

Les deux testicules de chaque sujet ont fait l'objet de mesures précises concernant le poids et le volume. Le volume est mesuré à l'aide d'un flacon gradué contenant, selon la taille de la pièce, 10 à $25 \mathrm{ml}$ de sérum physiologique. Le poids est calculé en soustrayant la tare, après avoir pesé l'ensemble : flacon, pièce et quantité de sérum.

\section{Etude microscopique}

Tous les prélèvements ont été réalisés selon la même orientation ; trois fragments ont été prélevés sur chaque testicule, un fragment hilaire, un fragment cortical et un fragment central.

Après fixation dans le liquide de Bouin, inclusion en paraffine et section de $7 \mu \mathrm{m}$, les coupes ont été colorées au trichrome de Masson, ce qui permet d'identifier clairement les différentes structures selon leur affinité tinctoriale.

\section{Etude morphométrique semi-quantitative}

Elle a été réalisée à l'aide d'un analyseur d'image. L'analyse a été pratiquée au grossissement X 400 .

Les deux logiciels utilisés sont : Adobe Photoshop 5.5 pour l'acquisition des images et, N.I.H. image (Scion image) pour le calcul des épaisseurs, diamètres et surfaces.

Outre l'épaisseur de l'albuginée $(\mu \mathrm{m})$ qui a été mesurée, concernant les tubes séminifères, cinq sections transversales de tubes séminifères ont été analysées par testicule; les paramètres étudiés sont : le diamètre de chaque tube $(\mu \mathrm{m})$ évalué sur les tubes coupés transversalement, la surface de chaque tube $\left(\mu \mathrm{m}^{2}\right)$, la surface totale occupée par les tubes dans un champ de surface donnée $\left(\mu \mathrm{m}^{2}\right)$, et l'épaisseur de la membrane propre $(\mu \mathrm{m})$.

Le comptage des cellules a été réalisé manuellement, après impression des images, pour les cellules de Sertoli, les spermatogonies, les spermatocytes I (pachytène), les spermatides, et les spermatozoïdes.
Concernant les cellules de Leydig, ont été mesurés : le nombre de groupements cellulaires par champ, la densité en cellules de Leydig par surface de champ, la surface du groupement cellulaire permettant d'évaluer le pourcentage de la surface occupée par les cellules de Leydig par rapport à la surface occupée par les tubes, et le diamètre de chaque cellule $(\mu \mathrm{m})$.

Six champs ont été étudiés sur chaque lame et six lames ont été examinées pour chaque fragment (hilaire, cortical et central).

\section{RESULTATS}

\section{Etude macroscopique}

Elle a montré une grande variabilité entre les sujets étudiés, mais globalement, le poids et le volume testiculaires chutent progressivement entre l'âge de 53 ans et l'âge de 102 ans (Figure 1).

\section{Analyses histologiques}

Les résultats histologiques ont montré plusieurs types d'altérations dont nous illustrons ici quelques aspects. L'albuginée peut présenter un épaississement fibreux en rapport avec l'âge (Figure 2A). L'un des signes fréquents est la sclérose artériolaire. Des collapsus des capillaires apparaissent, ce qui entraine leur raréfaction (Figure 2B). On note également une induration des espaces inter tubulaires due à une prolifération du tissu conjonctif, avec formation excessive de collagène accompagnée d'une hyalinose inter tubulaire (Figure 2B, Figure 2C). Les cellules de Leydig semblent parfois augmenter en nombre, formant de grands amas dans les espaces inter tubulaires (Figure 2D). En ce qui concerne l'histologie des tubes séminifères, la membrane propre s'épaissit, présentant parfois des hernies pouvant aller jusqu'à la disparition complète de l'épithélium séminifère, contribuant ainsi à la diminution du diamètre des tubes (Figure 2E). Les cellules de Sertoli se chargent de gouttelettes lipidiques et sont les dernières à disparaitre (Figure 2B). Les cellules de la lignée se raréfient, l'arrêt au stade spermatocyte I (pachytène) est le plus souvent observé (Figure 2F). L'aspect histologique le plus fréquent est constitué par une mosaïque de lésions différentes au niveau d'un même testicule. Tous ces aspects ont été quantifiés dans l'étude morphométrique.

\section{Etude morphométrique}

Elle confirme les données histologiques et montre que les différents paramètres étudiés sont perturbés par le vieillissement par rapport aux données obtenues chez des hommes plus jeunes (Tableau 1). La moyenne des diamètres des tubes séminifères diminue avec l'âge (Figure 3). L'épaisseur de la membrane propre augmente avec l'âge. Les 


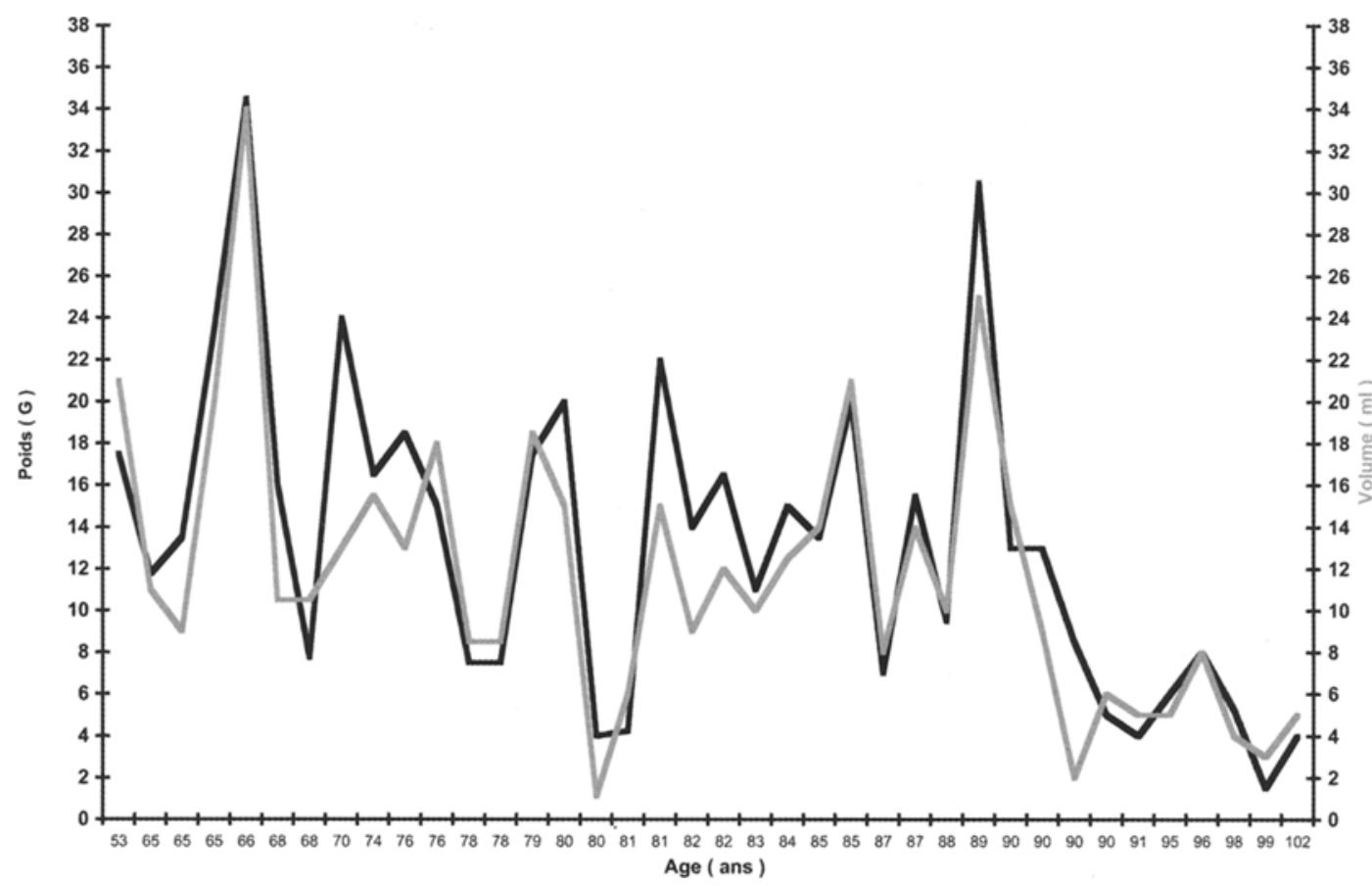

Figure 1 : Etude macroscopique : poids et volume testiculaires moyens mesurés chez 39 sujets décédés entre 53 et 102 ans.
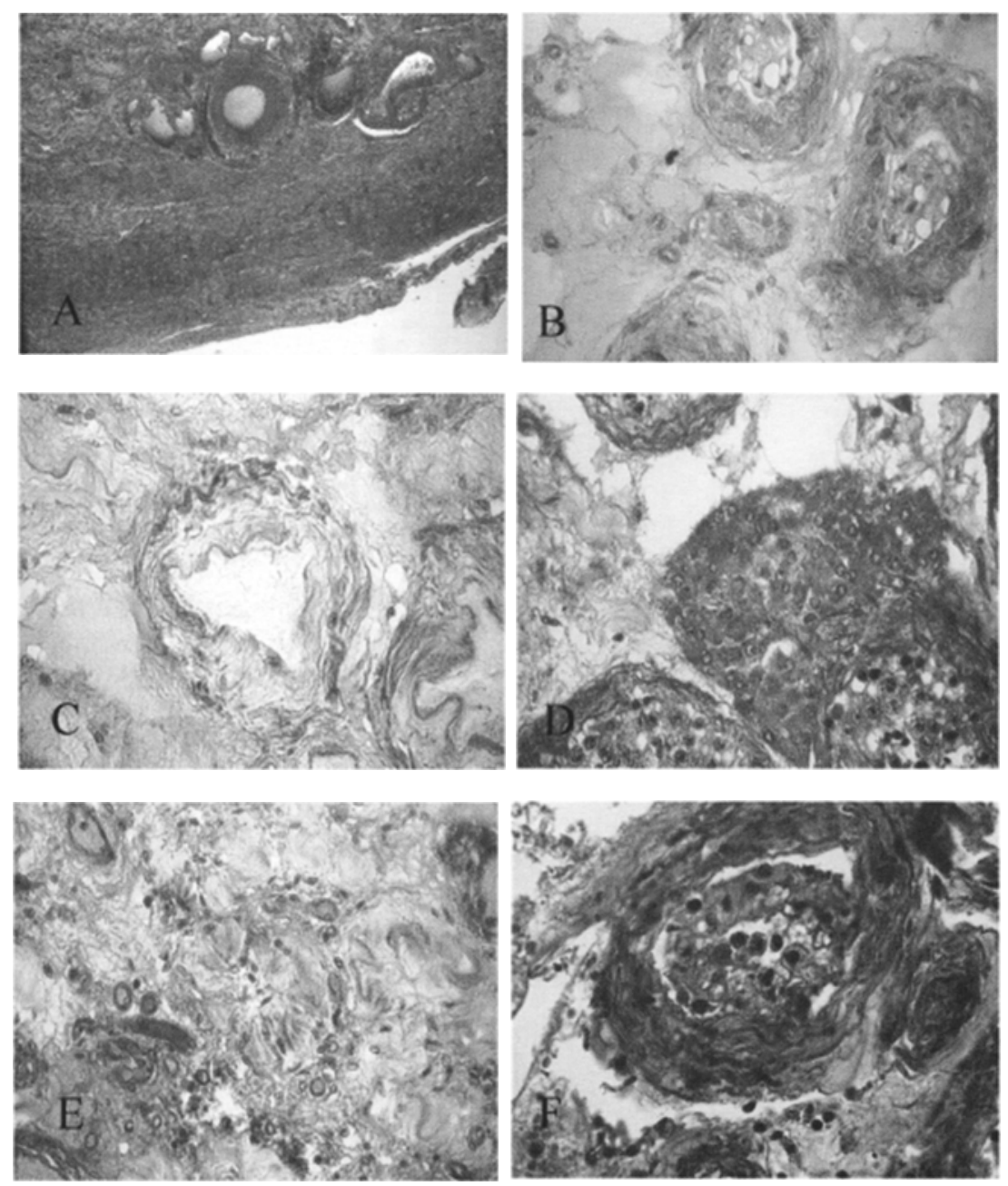

Figure 2 : Exemples d'altérations histologiques observées sur des coupes de testicules de sujets décédés âgés. Observation au grossissement $G X$ 400, coloration au trichrome de Masson.

2 A : Epaississement de l'albuginée observé chez un sujet décédé à 82 ans.

2 B : Raréfaction des capillaires et cellules de Sertoli présentant des gouttelettes lipidiques, observée chez un sujet décédé à l'âge de 82 ans.

2 C: Hyalinose inter tubulaire, tube séminifêre vide et membrane propre épaissie avec de nombreuses hernies, observés chez un sujet décédé à l'âge de 80 ans.

2 D : Amas de cellules de Leydig observé chez un sujet décédé à l'âge de 77 ans.

$2 E$ : Epaississement de la membrane propre présentant des hernies, disparition de l'épithélium et disparition de la lumière du tube, observés chez un sujet décédé à l'âge de 81 ans.

$2 F$ : Arrêt en pachytène observé chez un sujet décédé à l'âge de 89 ans. 


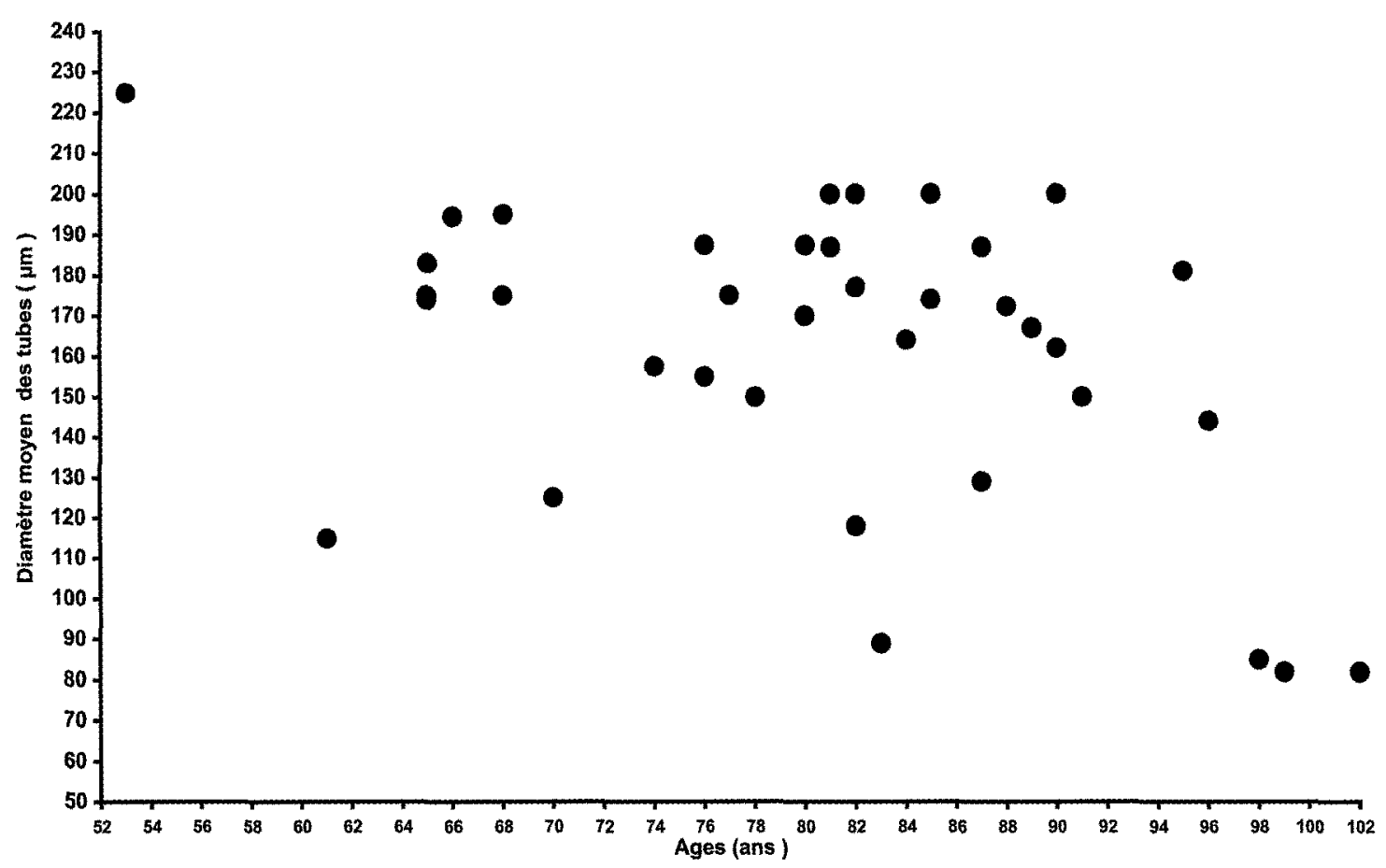

Figure 3 : Diamètre moyen des tubes séminifères en fonction de l'âge, mesuré à partir de sections transversales sur chaque testicule, chez 39 sujets décédés entre 53 et 102 ans.

Tableau 1 : Etude morphométrique de l'histologie testiculaire réalisée à partir de biopsies pratiquées chez 8 sujets témoins, présentant une agénésie bilatérale des canaux déférents et âgés entre 29 et 42 ans. Paramètres mesurés sur cinq sections transversales de tubes séminiferes par testicule (Valeur moyenne +/- écart type).

\begin{tabular}{lll}
\hline Tube séminifère & moyenne & écart-type \\
\hline Diamètre moyen des tubes $(\mu \mathrm{m})$ & 192,0 & $+/-0,8$ \\
Epaisseur de la membrane propre $(\mu \mathrm{m})$ & 5,3 & $+/-1,1$ \\
Nombre de cellules de Sertoli & 10,4 & $+/-2,6$ \\
Nombre de spermatogonies & 21,0 & $+/-4,2$ \\
Nombre de spermatocytes I & 31,0 & $+/-5,9$ \\
Nombre de spermatozoïdes & 25,0 & $+/-4,3$ \\
Cellules de Leydig & & \\
$\begin{array}{l}\text { Diamètre des cellules ( } \mu \text { m) } \\
\text { Rapport entre la surface occupée par } \\
\text { les cellules de Leydig et la surface } \\
\text { occupée par les tubes }\end{array}$ & 17,5 & $+/-2,5$ \\
& 3,1 & $+/-1,0$ \\
\hline
\end{tabular}

échantillons présentant une spermatogenèse complète ont tous une membrane propre d'épaisseur faible ; par contre dès qu'elle dépasse 10 microns (Figure 4), cela s'associe dans presque tous les cas soit à un arrêt de la spermatogenèse, soit à une fibrose complète. L'appauvrissement de l'épithélium séminifère est caractérisé par une diminution progressive du nombre de cellules de Sertoli (Figure 5).
Les lignées sont de plus en plus incomplètes au fur et à mesure que l'âge augmente. Seuls 19 sujets (environ la moitié) présentent encore des spermatozoïdes dans les tubes séminifères (Figure 6). Cependant, chez un homme âgé de 84 ans il persiste une spermatogenèse normale, et chez un autre sujet âgé de 95 ans, les tubes séminifères montrent encore la présence de quelques spermatozoïdes. La raréfaction des spermatozoïdes est en relation avec la diminution des spermatogonies (Figure 7) et des spermatocytes dont le nombre diminue avec l'âge (Figure 8). L'arrêt de la spermatogenèse se produit le plus souvent au stade pachytène. Les cellules de Leydig s'hypertrophient avec l'âge (Figure 9), puis semblent dégénérer au-delà de 90 ans. La richesse en cellules de Leydig estimée par la surface relative occupée, est le plus souvent augmentée par rapport aux témoins. Ce n'est qu'au-delà de 90 ans que ces cellules finissent par disparaître dans 3 cas sur 10 (Figure 10).

\section{DISCUSSION}

L'observation du vieillissement testiculaire dans notre série, montre différentes altérations déjà rapportées dans la littérature, mais que nous confirmons et quantifions. Habituellement, il est observé que la taille et le volume moyens des deux testicules sont peu modifiés avec l'âge [3], sauf en cas de fibrose et de hyalinose de tout le parenchyme. Nos résultats montrent un abaissement progressif dès l'âge de 60 ans malgré des variations individuelles importantes. Dans les espaces inter-tubulaires, des déformations des 


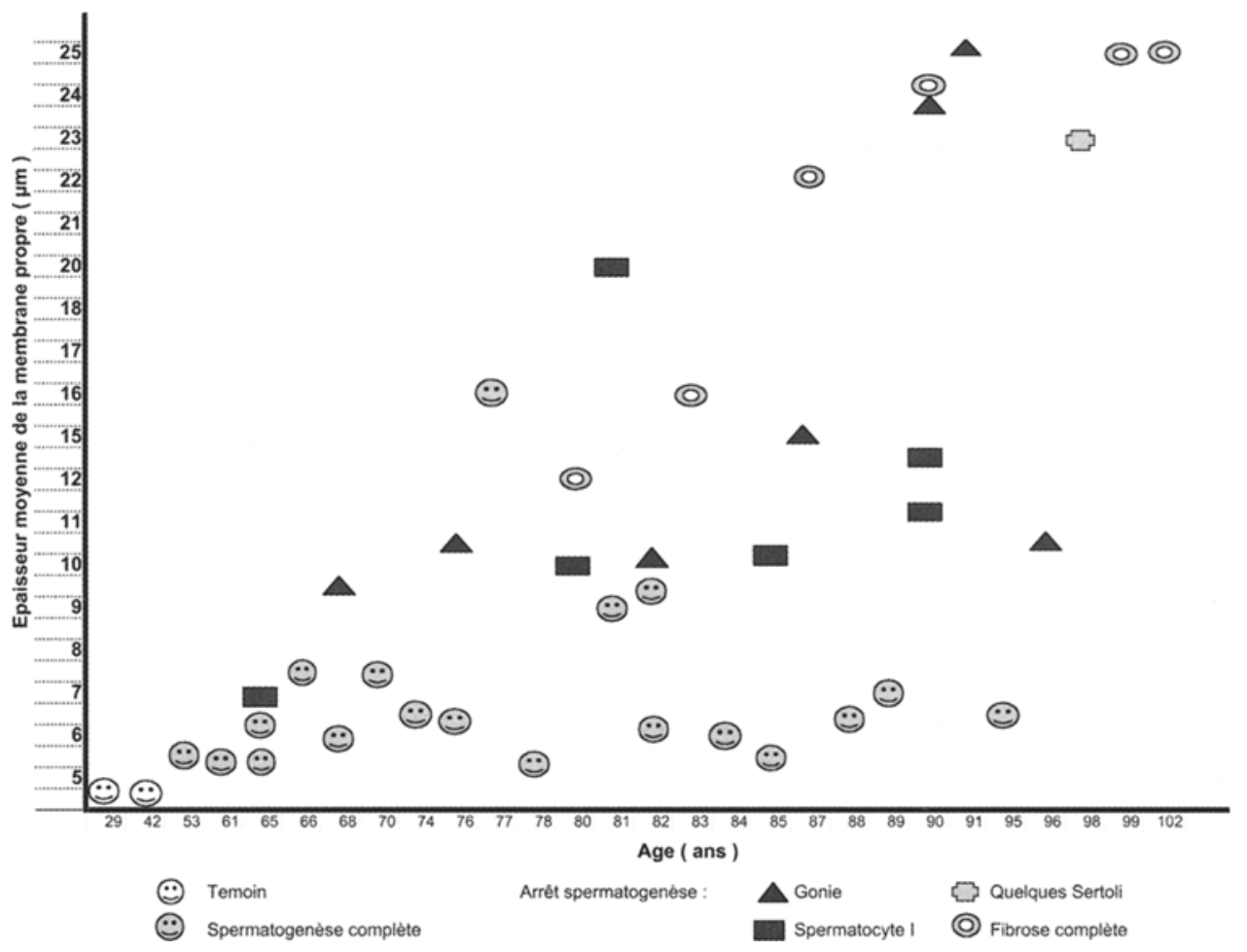

Figure 4 : Epaisseur de la membrane propre en fonction de l'âge en relation avec l'analyse histologique de la spermatogenèse chez 39 sujets décédés entre 53 et 102 ans.

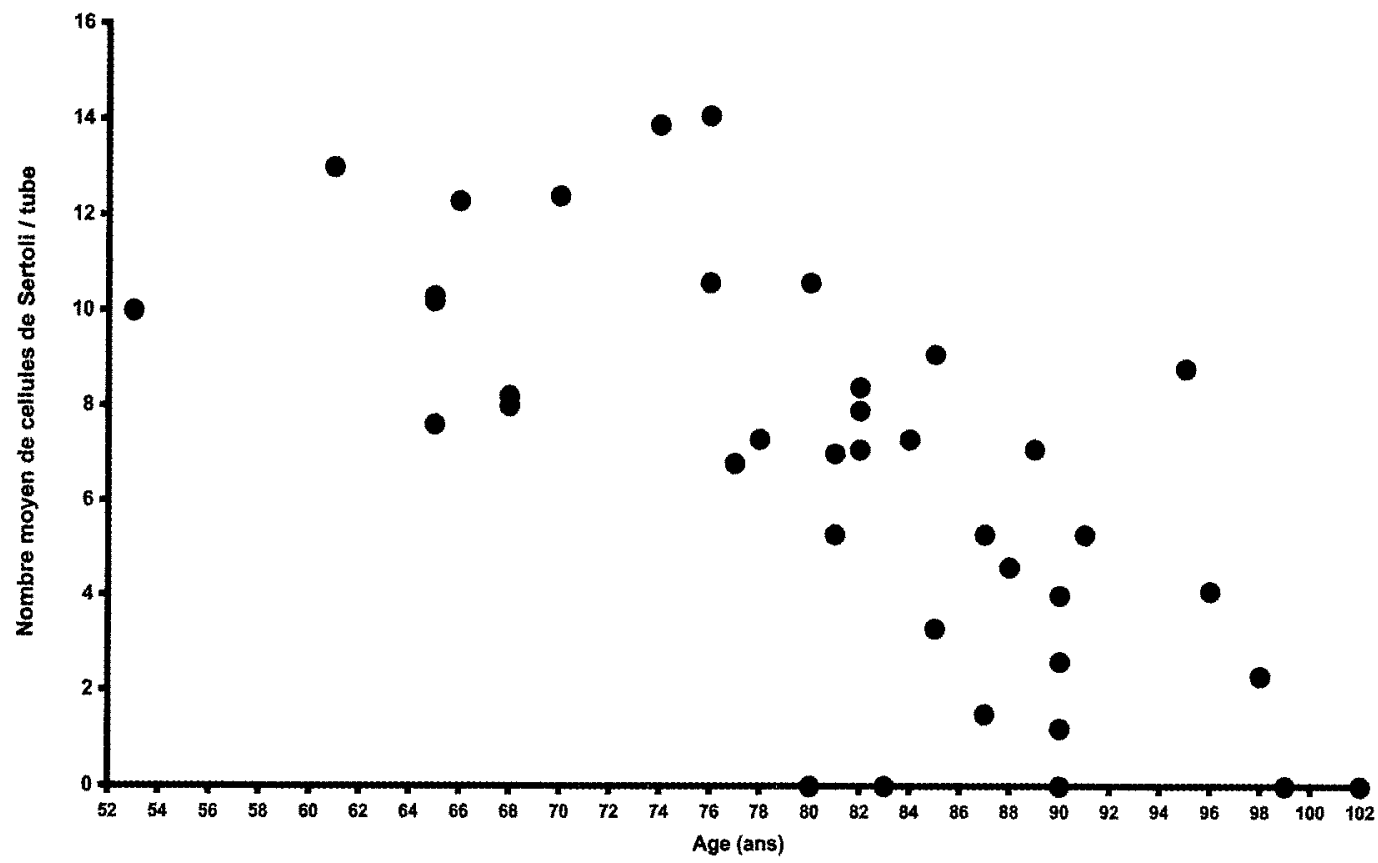

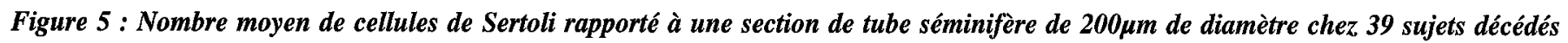
entre 53 et 102 ans. 


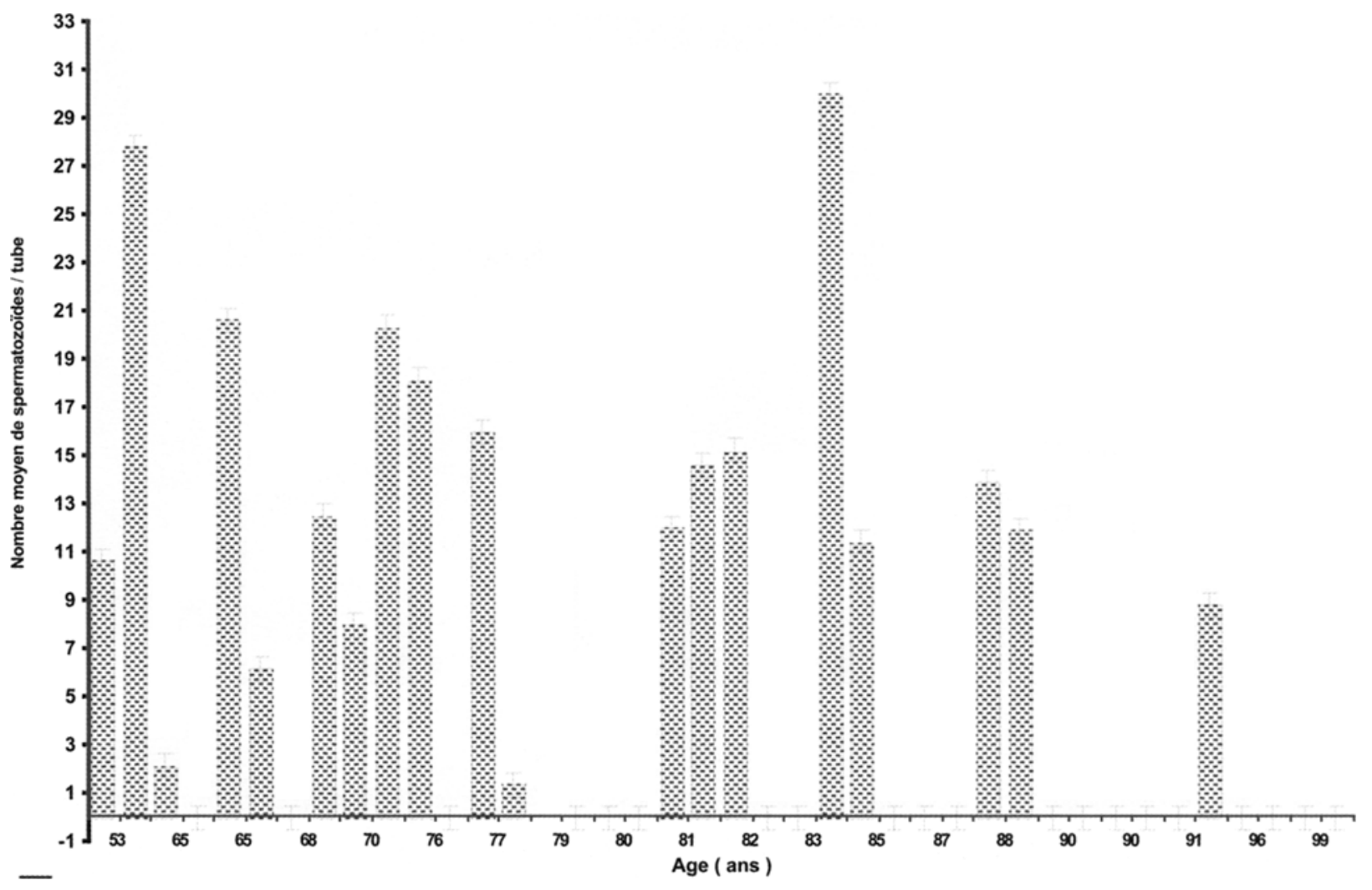

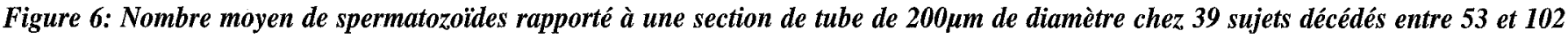
ans.

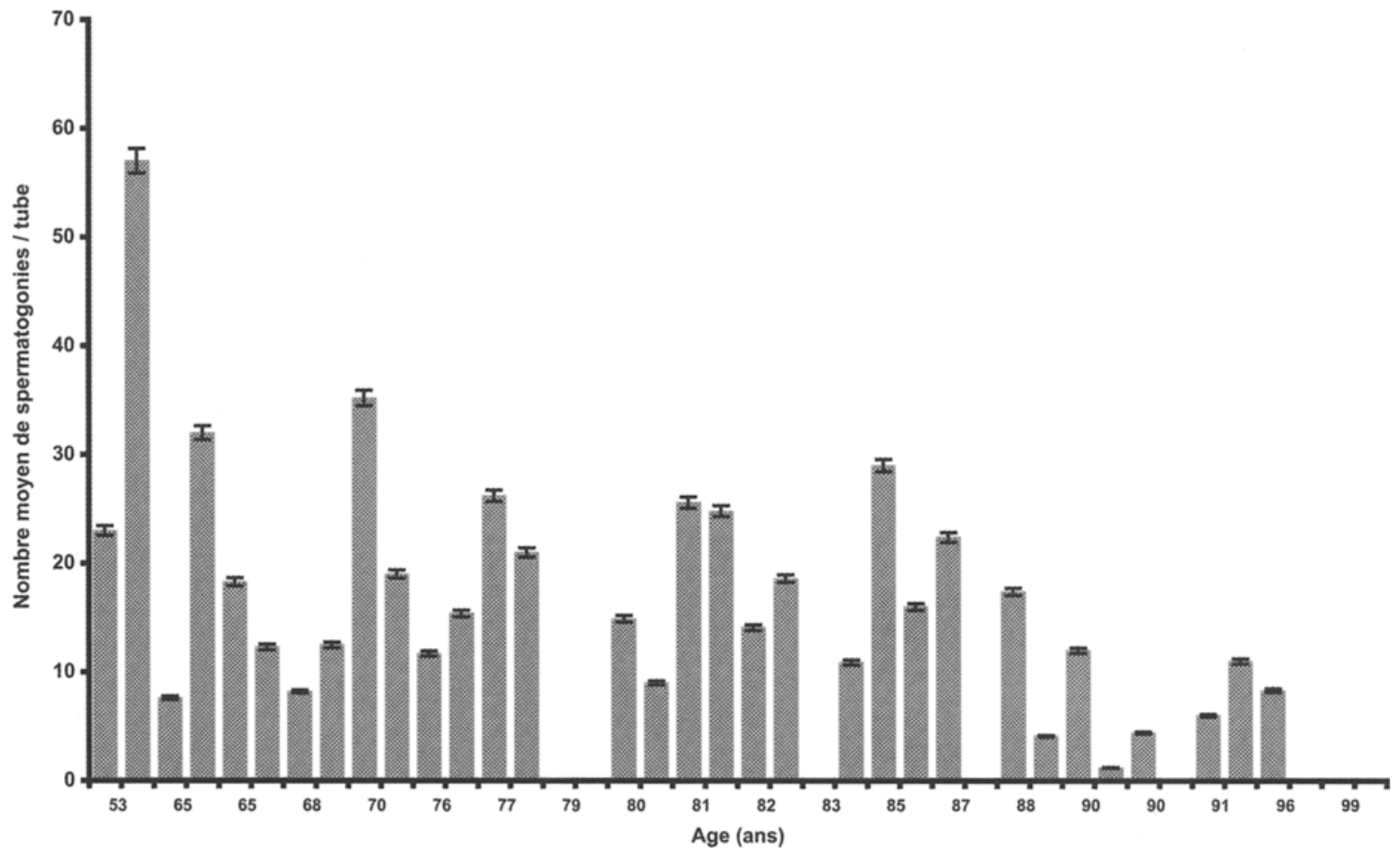

Figure 7 : Nombre moyen de spermatogonies rapporté à une section de tube de $200 \mu m$ de diamètre chez 39 sujets décédés entre 53 et 102 ans. 


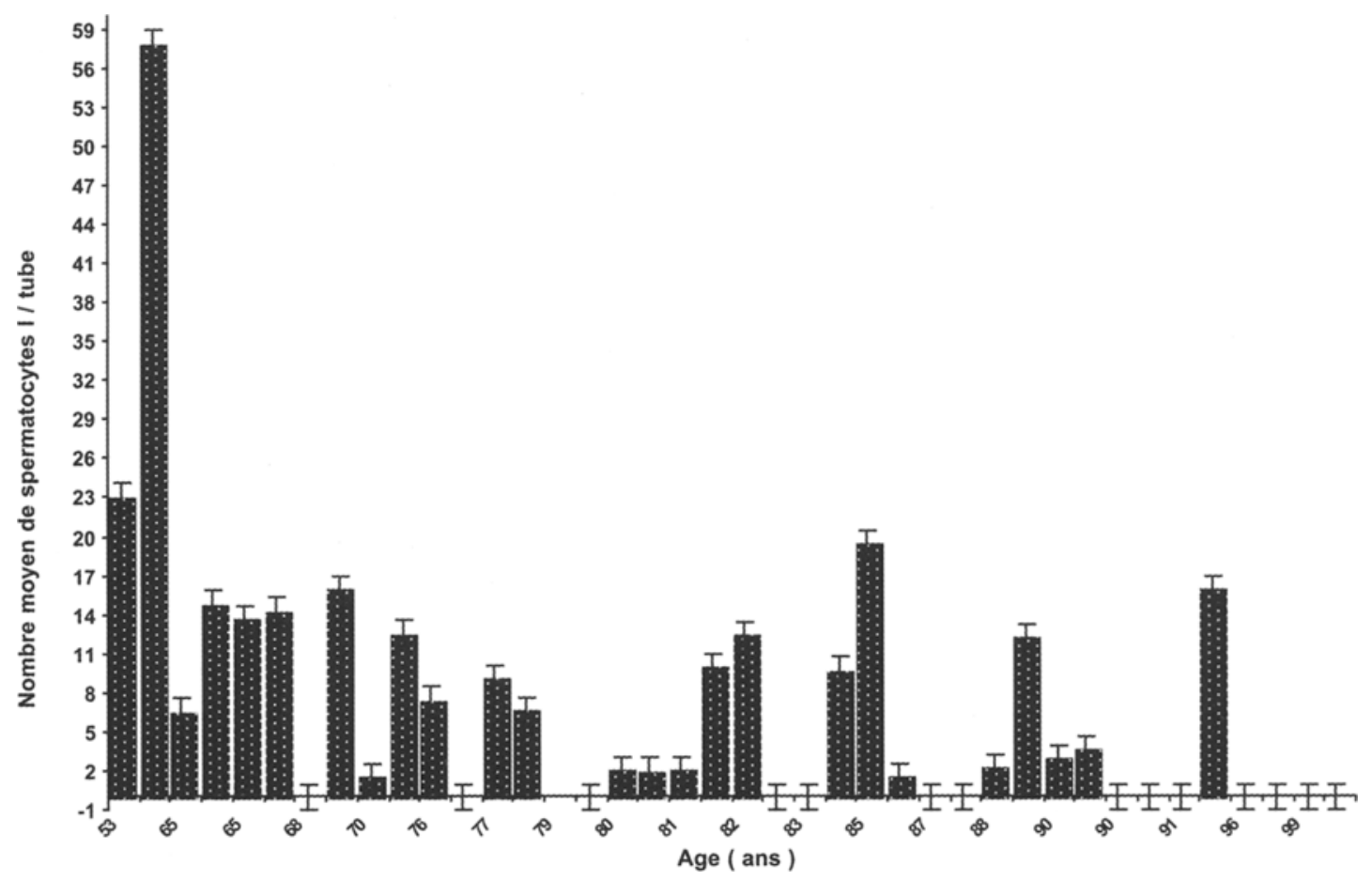

Figure 8 : Nombre moyen de spermatocytes I rapporté à une section de tube de $200 \mu m$ de diamètre chez 39 sujets décédés entre 53 et 102 ans.

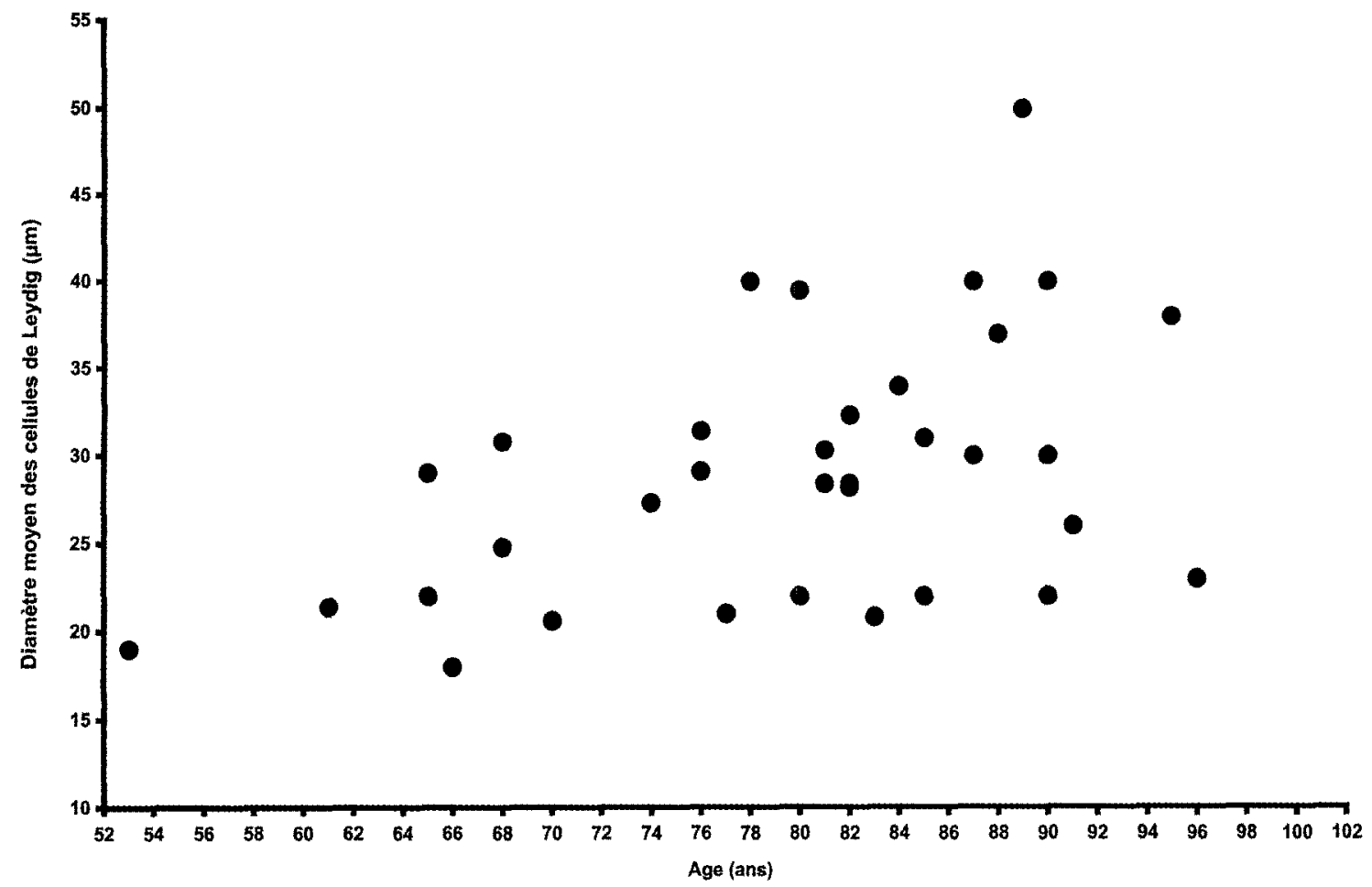

Figure 9 : Diamètre moyen des cellules de Leydig chez 39 sujets décédés entre 53 et 102 ans. 


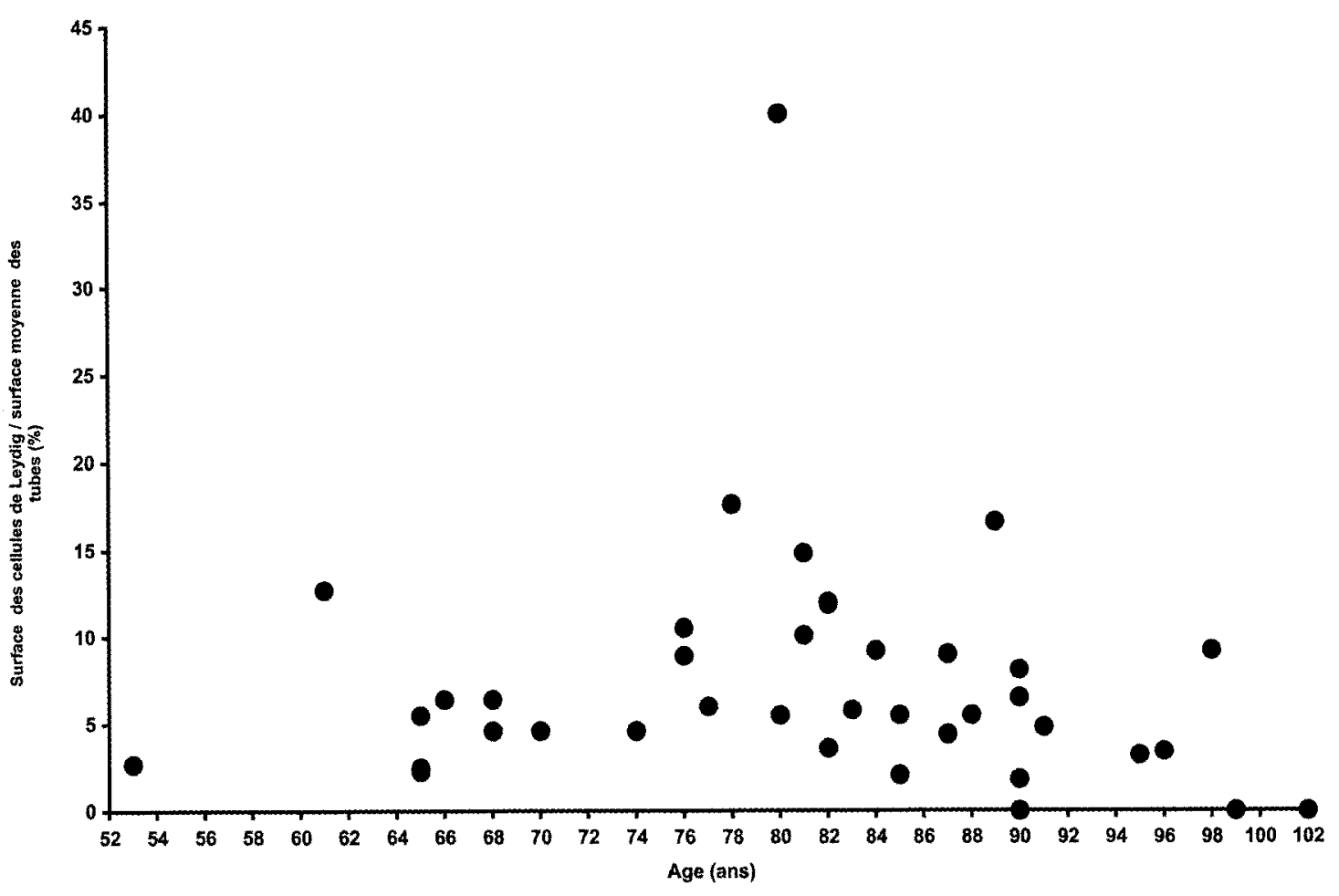

Figure 10 : Rapport entre la surface occupée par les cellules de Leydig et la surface moyenne occupée par les tubes chez 39 sujets décédés entre 53 et 102 ans.

artères inter-lobulaires sont décrites [23] ; des collapsus capillaires, suivis d'une raréfaction de ces derniers apparaissent avec l'âge [25]. Cette ischémie progressive entraîne une sénescence testiculaire. Ces caractéristiques ont toujours été constatées également dans notre étude au niveau de l'analyse histologique et expliquent certainement en partie les anomalies observées.

Au niveau du parenchyme testiculaire, la surface occupée par les tubes diminuerait avec l'âge [4], ce qui serait dû à une diminution du diamètre des tubes [18]. Cette constatation n'est pas tout à fait confirmée par nos mesures puisque le diamètre des tubes paraît stable jusqu'à 80 ans. Ce n'est que dans les cas de sclérose complète du tube avec disparition de l'épithélium séminifère et lorsque les espaces inter-tubulaires se chargent d'une fibro-hyalinose que le diamètre des tubes chute. Notre étude confirme que la membrane propre présente un épaississement progressif avec de nombreuses hernies, entraînant l'altération des tubes, puis l'atrophie complète après disparition de l'épithélium. L'épaississement débute dans notre série dès 60 ans et peut atteindre $25 \mu \mathrm{m}$. Ceci correspond le plus souvent à la sclérose des tubes. Lorsque la membrane reste fine, les tubes présentent une spermatogenèse complète.

Les cellules de Sertoli [13] montrent des modifications cytologiques au cours du vieillissement (surcharge lipidique, dilatation des espaces intercellulaires, plusieurs noyaux). Elles sont les dernières à disparaître [4]. Dans notre série, au delà de 75 ans, la population des cellules de Sertoli se raréfie et si cette diminution est retrouvée chez certains sujets, dans 5 cas on observe une disparition complète des cellules de Sertoli.

Les cellules de la lignée $[2,12]$ sont elles aussi très sensibles aux altérations testiculaires de l'homme âgé. Si pour certains la perte des cellules germinales détermine probablement les altérations des cellules de Sertoli [15], les perturbations observées sur les cellules de Sertoli peuvent directement retentir sur la production des spermatozoïdes [22]. Mais les résultats de nos observations montrent que ces deux phénomènes se produisent simultanément sans que l'on puisse déterminer quelle est la cause et quelle est la conséquence.

La disparition progressive des spermatogonies contribue à la disparition des spermatozoïdes [12]. L'arrêt de la spermatogenèse est cependant très fréquent au stade pachytène [4], qui est un stade de blocage fréquent de la spermatogenèse dans différentes situations d'altération du tissu testiculaire. Notre étude confirme ces résultats.

Les variations individuelles sont ici très importantes, puisqu'un sujet âgé de 84 ans présente un nombre de spermatozoïdes identique à celui des témoins. La spermatogenèse peut persister jusqu'à 95 ans alors que la disparition complète de la lignée peut débuter dès 65 ans.

Concernant les cellules de Leydig, les différentes études 
publiées donnent des résultats contradictoires. Si pour certains auteurs, la taille et le nombre des cellules de Leydig augmentent avec l'âge [8], d'autres estiment que leur nombre diminue avec l'âge, ou que cette diminution de nombre est compensée par une hypertrophie cellulaire [6]. Pour d'autres enfin, leur nombre reste constant, mais c'est un changement fonctionnel des cellules de Leydig [5] qui va de pair avec une baisse du taux de testostérone libre rencontrée chez $45 \%$ des sujets âgés $[7,11,16]$ et l'augmentation du taux de gonadotrophine plasmatique. Notre étude montre que la taille des cellules de Leydig est augmentée chez la plupart des sujets, la surface occupée évoluant peu. Par contre, chez certains sujets très âgés (plus de 90 ans) présentant une disparition complète de l'épithélium, on observe également un effondrement du nombre des cellules de Leydig. Du point de vue clinique, un éventuel traitement substitutif aux androgènes pourrait peut-être être discuté chez ces sujets.

L'existence de variations individuelles importantes est donc très claire dans nos résultats. Si certains sujets montrent d'importantes altérations dès 65 ans, la spermatogenèse peut être conservée à 95 ans. Cette variation peut avoir différentes causes (mode de vie, contact avec des toxiques, pathologies aiguës ou chroniques). Malheureusement, il est actuellement impossible de connaître les antécédents pathologiques, les traitements médicaux et les conditions environnementales des sujets ayant fait don de leur corps. Or tous ces facteurs externes pourraient expliquer certains cas d'altérations de la spermatogenèse. Cependant certains facteurs génétiques peuvent également être impliqués, prédisposant plus ou moins certains sujets à des altérations testiculaires plus importantes au cours du temps. Un autre aspect particulier décrit également par d'autres auteurs [17], est la coexistence d'altérations majeures dans certains tubes séminifères, alors que d'autres tubes ont un aspect normal. Ceci se retrouve aussi dans d'autres types d'altérations de la spermatogenèse et en particulier chez certains hommes infertiles. Cette hétérogénéité paraît être une des caractéristiques du tissu testiculaire.

\section{CONCLUSION}

Nos observations confirment les altérations de l'histologie testiculaire avec le vieillissement observé sur une série de 39 sujets âgés de 53 à 102 ans. Toutefois une grande hétérogénéité individuelle a été observée. Cette hétérogénéité est également observée à l'intérieur d'un même testicule. Nous avons ainsi pu montrer que la spermatogenèse peut rester conservée jusqu'à l'âge de 95 ans chez certains individus alors que dans d'autres cas de nombreuses altérations apparaissent dès 60 ans. Deux questions restent posées, celle des bases (génétiques et ou environnementales) de cette variabilité individuelle et celle des accidents génétiques [1] qui peuvent survenir au cours des méioses de ces hommes très âgés. La question de la paternité ne se pose sans doute plus à 95 ans, mais les demandes d'assistance médicale à la procréation émanant de couples dont le conjoint est âgé de plus de 60 ans ne sont plus exceptionnelles en 2002.

\section{REFERENCES}

1. AUROUX M.R. : Evolution of male fertility as a function of age and risks in progeny. Contracept. Fertil. Sex., 1991, 9 : 945-949.

2. HOLSTEIN A.F. : Spermatogenesis in old age, a borderland between normal and pathological anatomy. Urologe A, 1986, 25 : $130-137$.

3. JOHNSON L., PETTY C.S., NEAVES W.B. : Influence of age on sperm production and testicular weights in men. J. Reprod. Fertil., 1984, $70:$ 211-218.

4. JOHNSON L. : Spermatogenesis and aging in the human. J. Androl., 1986, 7 : 331-354.

5. KADEN R. : Testicular histology in the aging man. Fortschr. Med., 1975, $93: 1545-1549$.

6. KALER L.W., NEAVES W.B. : Attrition of the human Leydig cell population with advancing age. Anat. Rec., 1978, 192 : 513518 .

7. KAUFMAN J.M., VERMEULEN A. : Declining gonadal function in elderly men. Baillieres Clin. Endocrinal Metab., 1997, 11: 289-309.

8. KOTHARI L.K., GUPTA A.S. : Effect of ageing on the volume, structure and total Leydig cell content of the human testis. Int. J. Fertil., 1974, $19:$ 140-146.

9. MANIERI C., FORNENGO R., MOLINATTI G.M. : Male fertility in the elderly. Arch. Ital. Urol. Androl., 1993, 65 : 501-505.

10. NEAVES W.B., JOHNSON L., PORTER J.C.Jr. et al. : Leydig cells numbers, daily sperm production, and serum gonadotropin levels in aging men. J. Clin. Endocrinol. Metab., 1984, 59 :756763.

11. NEAVES W.B., JOHNSON L.; PETTY C.S. : Seminiferous tubules and daily sperm production in older adult men with varied numbers of Leydig cells. Biol. Reprod., 1987, 36 : 301308.

12. NISTAL M., CODESAL J., PANIAGUA R. et al. : Decrease in the number of human Ap and Ad spermatogonia and in the Ap/Ad ratio with advancing age. New data on the spermatogonial stem cell. J. Androl., 1987, 8 : 64-68.

13. PANIAGUA R., AMAT P., NISTAL M. et al. : Ultrastructural changes in Sertoli cells in ageing humans. Int. J. Androl., 1985, 8: $295-312$.

14. PANIAGUA R., AMAT P., NISTAL M. et al. : Ultrastructure of Leydig cells in human ageing testes. J. Anat., 1986, 146 : 173183.

15. PANIAGUA R., RODRIGUEZ M.C., NISTAL M et al. : Changes in the lipid inclusion/Sertoli cell cytoplasm area ratio during 
the cycle of the human seminiferous epithelium. J. Reprod. Fertil., 1987, $80: 335-341$.

16. PANIAGUA R., MARTIN A. NISTAL M. et al. : Testicular involution in elderly men: comparison of histologic quantitative studies with hormone patterns. Fertil. Steril., 1987, 47 : 671-679.

17. PANIAGUA R., NISTAL M., AMAT P. et al. : Seminiferous tubule involution in elderly men. Biol. Reprod., 1987, 36 : 939947.

18. PANIAGUA R., NISTAL M., SAEZ F.J. et al. : Ultrastructure of the aging human testis. J. Electron. Microsc. Tech., 1991, 19 : 241-260.

19. PLAS E., BERGER P., HERMANN M. et al. : Effects of aging on male fertility? Exp. Gerontol., 2000, 35 : 543-551.

20. REGADERA R., NISTAL M., PANIAGUA R. : Testis, epididymis, and spermatic cord in elderly men. Correlation of angiographic and histologic studies with systemic arteriosclerosis. Arch. Pathol. Lab. Med., 1985, 109 : 663-667.

21. REGADERA J., COBO P., MARTINEZ-GARCIA F. et al. : Testosterone immunoexpression in human Leydig cells of the tunica albuginea testis and spermatic cord. A quantitative study in normal foetuses, young adults, elderly men patients with cryptorchidism. Andrologia, 1993, $25: 115-122$.

22. RUSSELL L.D., PETERSON R.N. : Determination of elongate spermatid-Sertoli cell ratio in various mammals. J. Reprod. Fertil., 1984, $70: 635-641$.

23. SASANO N., ICHIJO S. : Vascular patterns of human testis with special reference to its senile changes. Tohoku J. Exp. Med., $1969,99: 269-280$.

24. SEYMOUR F., DUFFY C., KORNER A. : A case of authentic fertility in a man of 94. J. Am. Med. Ass., 1935, 105 : 14231424.

25. SUORANTA $\mathrm{H}$. : Changes in the small blood vessels of the adult human testis in relation to age and some pathological conditions. Virchows Arch., 1971, B 352 : 165-181.

\author{
ABSTRACT \\ Semiquantitative morphometric study of testicular \\ aging \\ Lucia BICCHIERAY, Imen BEN FTIMA, Martine \\ ALBERT, Marianne BERGERE, Olivier CUSSENOT, \\ Nicole PARSEGHIAN, Mbarka DAKOUANE, Jacqueline \\ SELVA.
}

Testicular aging is usually studied using sperm and quantitative hormone analysis. Testicular samples are obviously difficult to obtain from a control aging population. Body donations from the Anatomy Department of the Saint-Peres University provided access to testicular samples from deceased men between the ages of 53 to 102 years. We present the first results of a semiquantitative histological morphometric study of testicular aging. We studied a series of 39 subjects. After removal of the sample within the first 24 hours, several investigations were conducted. Macroscopic examination (volume, weight) was followed by histological examination and computer-assisted morphometric analysis: N.I.H images based on the following parameters: (i) transverse sections of the seminiferous tubules (total surface, thickness of the basal membrane, and nuclear density of Sertoli cells, spermatogonia, spermatocytes and spermatozoids; (ii) histological sections were studied for interstitial tissue, number of clusters and the surface occupied by Leydig cells (percentage per parenchyma area), their appearance, size and nuclear density were determined; (iii) this study was completed by visual count of the various cell types in the seminiferous epithelium.

The results obtained on a series of 39 subjects aged from 53 to 102 showed various alterations, such as thickening of the tunica albuginea and basal membrane and intertubule hyalinization. The most frequent histological pattern of the aging testis is a mosaic of various seminiferous tubule lesions varying from tubules with complete although reduced spermatogenesis to entirely sclerosed tubules. Individual variations are extremely marked with major alterations of spermatogenesis as early as $\mathbf{6 0}$ years old, with atrophied Leydig cells and, on the contrary, preserved spermatogenesis until the age of 95 years.

Key Words: germ cells, basal membrane, seminiferous epithelium, testicular aging 